\title{
Pontine demyelination as a late complication of resolved mild COVID-19 infection
}

\author{
Nouran Eshak MD, Mahmoud Abdelnabi MD, Roy Jacob MD, J. Drew Payne DO
}

\begin{abstract}
Previous case reports have demonstrated COVID-19 related neurotropism. Neural infection may result from trans-lamina cribrosa invasion that allows COVID-19 to reach the brain through the olfactory tract. A wide range of symptoms from headaches, anosmia, dysgeusia to neuropathy, encephalitis, cerebrovascular stroke, and rarely demyelination has been reported. Here, we report a case of pontine demyelination causing generalized weakness as a rare neurological complication in a COVID-19 survivor. Our case highlights that even mild and moderate COVID19 infection can have late neurological sequelae.
\end{abstract}

Keywords: COVID-19, demyelination, neurological complications, corticosteroids

\section{INTRODUCTION}

The COVID-19 global pandemic is caused by the severe acute respiratory syndrome coronavirus-2 (SARS CoV-2). The presentation usually involves upper respiratory symptoms, such as dry cough, dyspnea, and chest pain that can be complicated by respiratory failure and death. Several previous reports highlighted the neurotropism of COVID-19, even in mild and moderate cases. Neural invasion may occur through trans-lamina cribrosa that allows COVID-19 to reach the brain through the olfactory tract. ${ }^{1} \mathrm{~A}$ wide range of symptoms has been reported, ranging from headaches, anosmia, dysgeusia to neuropathy, encephalitis, cerebrovascular stroke, and rarely demyelination. ${ }^{2}$ Several immune-medicated mechanisms can explain early and late neurological sequelae related to COVID-19 infection Here, we report a case of pontine demyelination causing generalized weakness as a rare neurological complication in a COVID-19 survivor.

Corresponding author: Nouran Eshak

Contact Information: Nouran.Eshak@ttuhsc.edu

DOI: 10.12746/swrccc.v9i41.947

\section{CASE}

A 45-year-old male patient presented to our medical facility after experiencing a sudden episode of dizziness, slurred speech, and upper and lower extremity weakness and numbness that partially resolved within 24 hours. He had been experiencing mild generalized weakness for a month but did not seek medical attention. Past medical history was significant for uncontrolled diabetes mellitus, hypertension, and a resolved COVID-19 infection two months prior to this presentation. At the time of the COVID-19 infection, he had fever and shortness of breath but did not require supplemental $\mathrm{O} 2$ or hospitalization. A general physical examination and vital signs were normal. Neurological assessment showed mild generalized symmetrical weakness in upper and lower extremities with no facial involvement (muscle strength was $4+/ 5$ in all extremities); the rest of the examination was otherwise unremarkable. Laboratory tests, including CBC, metabolic panel, ESR, CRP, and nasopharyngeal swab for COVID-19, were normal. Computed tomography (CT) of the brain without contrast (Figure 1 Panel A) and CT angiography were unremarkable. Cerebrospinal fluid (CSF) studies were within normal limits; there were no oligo-clonal bands. Bacterial and fungal antigens 

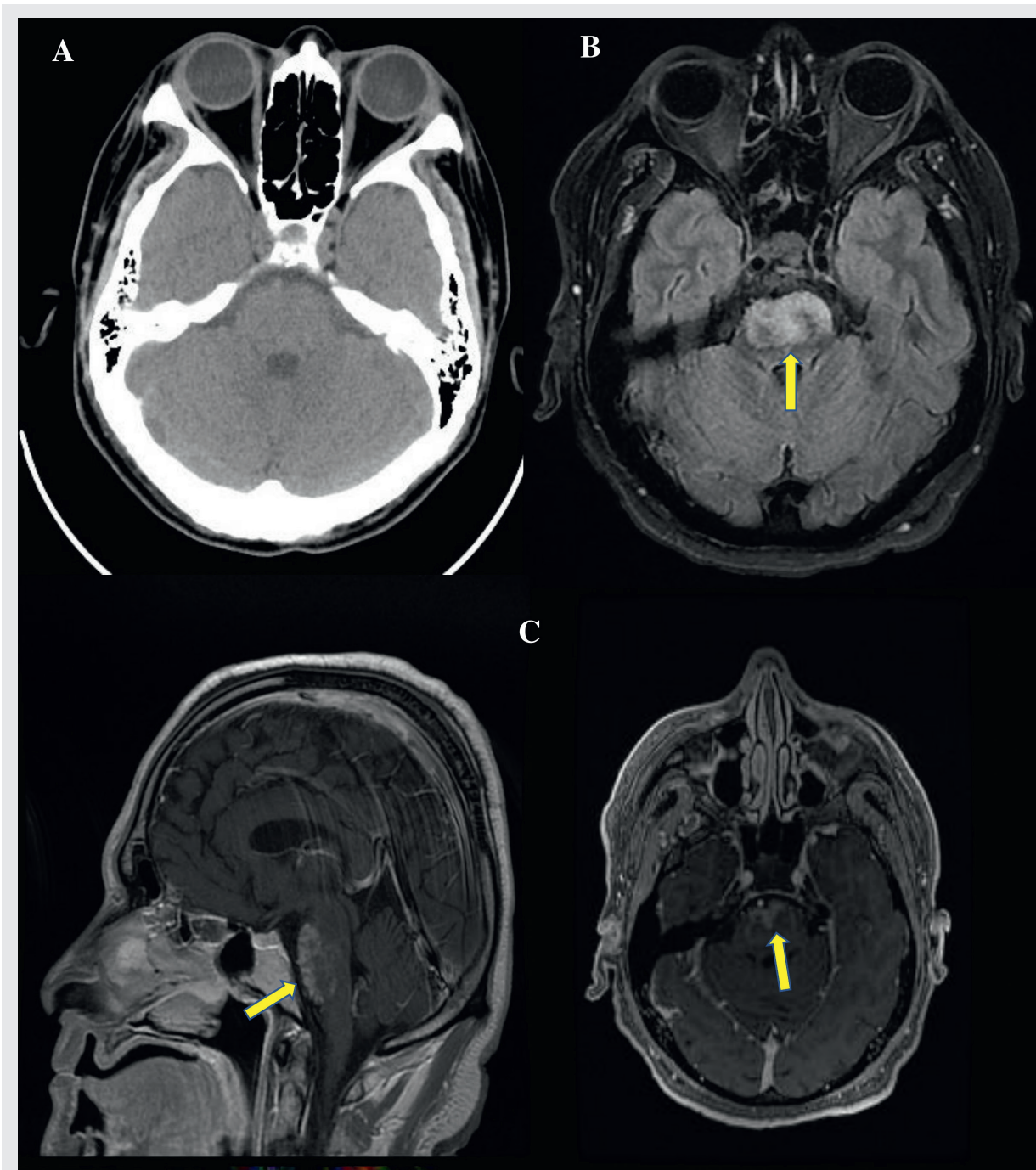

D

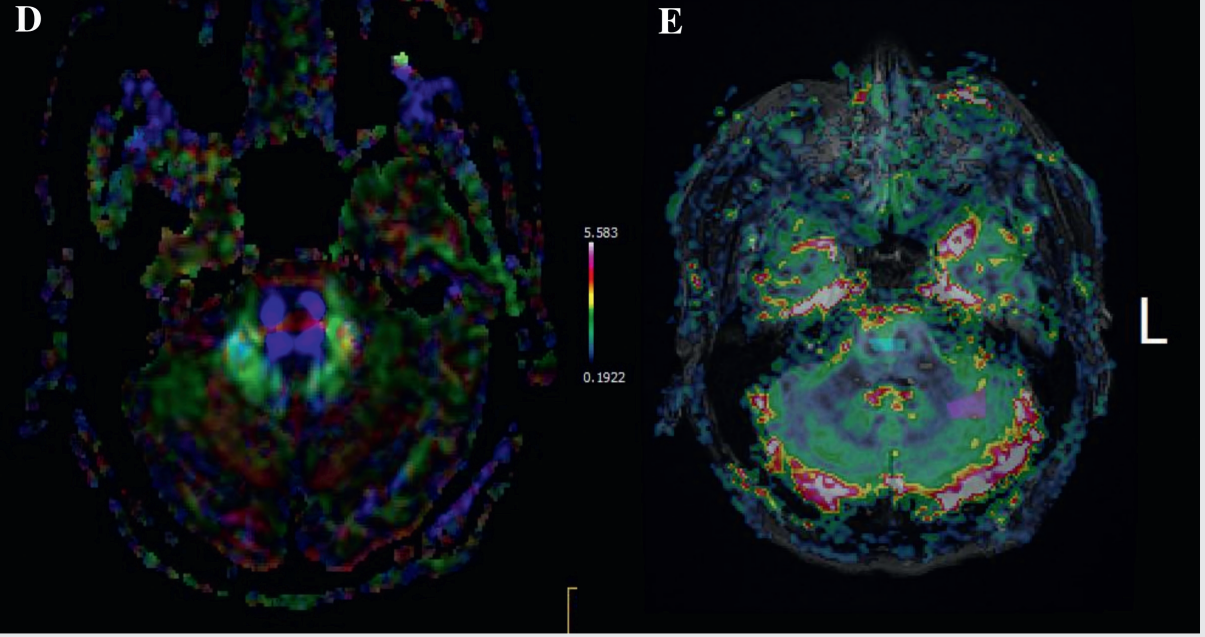

Figure 1. Panel A: CT brain without contrast showing no abnormality in the region of the pons. Panel B: Axial T2 FLAIR MRI image demonstrating abnormal increased T2 FLAIR signal within the ventral pons. (Arrow) Panel C: Axial T1 postcontrast image and sagittal $\mathrm{T} 1$ postcontrast image showing abnormal lacelike enhancement in the pons. (Arrow) Panel D: MRI Diffusion tensor imaging demonstrating normal transverse pontine fibers and corticospinal tracts within the pons with no significant mass effect. Panel E: MRI perfusion with dynamic susceptibility contrast imaging technique showing no increased cerebral blood flow within the lesion in the pons. 


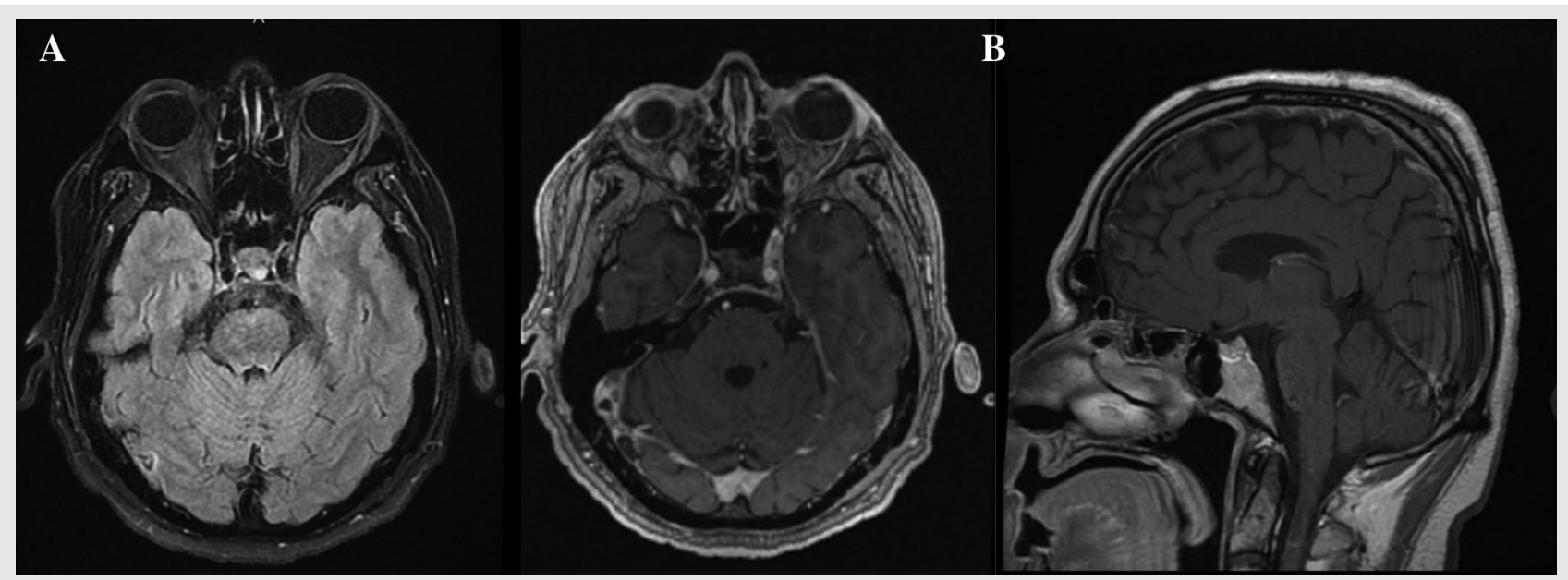

Figure 2. Panel A: Axial T2 FLAIR MRI image demonstrating improvement of the previously present abnormal increased T2 FLAIR signal within the ventral pons. Panel B: Axial T1 postcontrast image and sagittal T1 postcontrast image showing resolution of the previously seen lacelike enhancement.

and CSF RT-PCR for neurotropic viruses, including COVID-19, were negative. Magnetic resonance imaging (MRI) of the brain and spine with diffusion showed an increased T2 flair signal with lacelike enhancement within the pons (Figure 1, Panel B-E), affecting corticospinal tracks suggesting a diagnosis of post-infection pontine demyelination.

He was started on methylprednisolone $500 \mathrm{mg}$ IV for five days with a gradual improvement of his weakness and discharged with a plan to taper the corticosteroids and to repeat an outpatient MRI. Follow-up MRI brain after two months showed improvement of hyperintensity and resolution of previously seen lacelike enhancement in the pons (Figure 2).

\section{Discussion}

Neurological involvement in COVID-19 is quite common; Mao et al. ${ }^{2}$ reported that more than one-third of COVID-19 patients develop neurological manifestations. Symptoms have ranged from headache, anosmia, and dysgeusia to neuropathy, encephalitis, cerebrovascular stroke, and rarely Guillan-Barrè syndrome (GBS) ${ }^{3}$ and demyelination. ${ }^{4}$ COVID-19 induced delayed CNS damage could be immune-mediated through several proposed mechanisms. A virus-induced systemic inflammatory response (SIRS) or SIRS-like immune disorders with a resultant pro-inflammatory state with IL1, IL-6, and TNF $\alpha$ release could cause glial cell activation with subsequent demyelination. Another possible mechanism might be the production of antibodies against glial cells triggered by viral infection. ${ }^{4}$ Previous studies on SARS-CoV-1 showed viral replication leads to delayed self-reactive T-cell suppression resulting in neuroinflammation and central nervous system (CNS) demyelination or axonal damage of the CNS. ${ }^{5}$ Recent studies have shown that COVID-19 can cross the blood-brain barrier and cause acute or delayed CNS demyelination or axonal damage. ${ }^{1}$

In contrast to this case report in which the patient presented with pontine demyelination as a late neurological complication of COVID-19, several neurological presentations have been reported during the acute phase of COVID-19 infection, including extra pontine myelinolysis, ${ }^{6}$ acute disseminated encephalomyelitis (ADEM), ${ }^{7}$ and necrotizing encephalitis. A high index of suspicion and prompt treatment with immune-modulatory therapies, such as IVIG or pulse steroids, should be considered to avoid permanent neural damage.

\section{Conclusion}

Unlike previously reported cases that linked neurological complications to severe COVID-19 infections, 
our case presented with weakness due to post-infectious demyelination after a mild COVID-19 infection. This indicates that even mild and moderate COVID-19 infection can have late neurological sequelae.

Article citation: Eshak N, Abdelnabi M, Jacob R, Payne JD. Pontine demyelination as a late complication of resolved mild COVID-19 infection. The Southwest Respiratory and Critical Care Chronicles 2021;9(41):60-63 From: Department of Internal Medicine (NE, MA, JDP), Texas Tech University Health Sciences Center, Lubbock, Texas; Clinical and Experimental Internal Medicine Department (MA), Medical Research Institute, Alexandria University, Alexandria, Egypt; University Medical Center, Radiology Department (RJ), Lubbock, Texas

Submitted: 9/23/2021

Accepted: 10/12/2021

Reviewer: Parunyou Julayanont MD

Conflicts of interest: none

This work is licensed under a Creative Commons

Attribution-ShareAlike 4.0 International License.

\section{REFERENCES}

1. Desforges M, Le Coupanec A, Dubeau P, et al. Human coronaviruses and other respiratory viruses: underestimated opportunistic pathogens of the central nervous system? Viruses 2020;12(1):14.

2. Mao L, Jin H, Wang M, et al. Neurologic manifestations of hospitalized patients with coronavirus disease 2019 in Wuhan, China. JAMA Neurology 2020;77(6):683-690.

3. Toscano G, Palmerini F, Ravaglia S, et al. Guillain-Barré syndrome associated with SARS-CoV-2. N Engl J Med 2020; 382:2574-2576.

4. Zanin L, Saraceno G, Panciani PP, et al. SARS-CoV-2 can induce brain and spine demyelinating lesions. Acta Neurochirurgica 2020:1-4.

5. Cheng Y, Skinner DD, Lane TE. Innate immune responses and viral-induced neurologic disease. J Clinical Medicine 2019; $8(1): 3$.

6. Abd Ur Rehman M, Abdulrahman AF, Zainab A, et al. Hyponatremia and extrapontine myelinolysis in a patient with COVID 19: A case report. Clinical Case Reports 2021;9(7): e04463.

7. Parsons T, Banks S, Bae C, et al. COVID-19-associated acute disseminated encephalomyelitis (ADEM). J Neurology 2020; 267:2799-2802. 